\title{
perCues: Trails of Persuasion for Ambient Intelligence
}

\author{
Manfred Tscheligi, Wolfgang Reitberger, Christoph Obermair, Bernd Ploderer, \\ HCI\&Usability Unit, ICT\&S Center, University of Salzburg, Sigmund-Haffner-Gasse 18, \\ 5020 Salzburg, Austria \\ \{manfred.tscheligi, wolfgang.reitberger, christoph.obermair, \\ bernd.ploderer\}@sbg.ac.at \\ http://www.icts.sbg.ac.at/
}

\begin{abstract}
The realization of the ambient intelligence (AmI) vision will have a profound impact on our everyday lives and society. AmI applied in contexts like homes or public spaces will not only affect individual users but influence entire groups of users. The question is how we can apply such technologies to persuade groups and individual users. Our approach is to design AmI environments by borrowing a concept which works very well in biological and social systems: Collective Intelligence (CI). The intelligence of a group surpasses the individual intelligences and leads to improved problem solving capabilities of individuals and groups. From nature we borrow examples of cues in the environment to stimulate goal directed collective intelligence (perCues). The application of perCues in AmI environments helps to persuade users to reach a common goal like decreasing environmental pollution. Adopting CI for AmI we blaze a trail for the design of persuasive AmI environments.
\end{abstract}

\section{Introduction}

Ambient Intelligence (AmI) emerged from the developments of ubiquitous computing [1], natural interaction and intelligent systems. The AmI vision describes the pervasion of the everyday world with digital technology which is able to anticipate the user's needs and to support the user in fulfilling these needs [2]. AmI adapts to the requirements of the users and reacts to their presence.

The goal of this paper is to blaze a trail for the use of AmI environments to positively influence and persuade groups and individual users. Moreover these environments aim to increase the users' problem-solving capabilities. Our approach towards persuasion in AmI is informed by the concept of Collective Intelligence (CI). Humans can solve problems more efficiently together than alone as shown in social psychology research [3] demonstrating the phenomenon of collective intelligence. AmI goes beyond the classical single user - computer interface and allows us to involve a larger number of people. Examples of $\mathrm{CI}$ in nature show us efficient ways for the design of AmI environments fostering CI among humans. In this way we aim to persuade people to behave in a sustainable manner. 


\section{Collective Intelligence}

Collective Intelligence names the increased problem-solving capacity that results from synergetic effects of interacting intelligent agents [4;5]. CI leads to cooperation between individuals, but not to collectivization, there is a delicate balance between individuality and relations (collective). CI is a form of universal distributed intelligence which is constantly enhanced and coordinated in real time. Levy also stresses the use of appropriate communication structures for the coordination of CI in real time [5]. These structures can also be built with digital communication technologies. CI improves the problem solving capability of a system since a group of people has better problem solving capabilities that it's individual members alone.

Related approaches in HCI are distributed cognition [6] and social intelligence [7]. Both approaches are relevant to CI, however CI does not only describe the relations between individuals but can be described as a goal for a group to be reached. Only under certain circumstances can individuals profit from CI leading to increased problem solving capabilities.

\subsection{Collective Intelligence in Natural Systems}

In some cases, the evolution has combined hundreds and thousands of individual brains to higher entities. Ants, bees (and also other insects) display collectively intelligent behavior when foraging for food or fighting off predators. Each ant in the colony acts in a rather simple way, but together they end up doing something intelligent, like discovering the shortest path between their ant hill and a food source.

In a bee colony no individual bee knows about the geographic distribution of all food sources in the area. It decides based on the food requirements of the entire colony if a certain food source is worth being exploited. A single bee is not informed about a global measurement like the food requirements of the colony, but it can deduct this in-formation from a local cue in the environment: the length of the queue at the hive [8].

\subsection{Ambient Intelligence Informed by Collective Intelligence}

CI has been applied to the cyberspace [5], to multi-agent systems, and artificial intelligence [9]. Shirvanee and Davenport [10] used CI for a better understanding of social experiences in public spaces. Our approach is to adopt the concept of CI and especially particular cues in the environment for persuasive AmI environments. We propose the concept of perCues to describe ambient cues towards persuasion. perCues present visual stimuli in the environment (ambient displays) aiming to inform individuals and groups and to encourage a certain behavior. perCues inherit properties from environmental cues in nature providing feedback about a certain aspect of the collective state guiding the individual behavior. AmI environments with perCues foster CI and persuade people to behave in a sustainable manner. 


\section{3 perCues for Sustainable Mobility}

We are currently investigating the potentials of perCues applied on ambient displays in public spaces to persuade people to behave in a collectively intelligent sustainable way. The Kyoto report of Salzburg states that one third of the carbon dioxide emissions in the city are caused by traffic. Thus the authors propose to shift traffic to public mass transport [11]. One concrete scenario to reach this goal is to persuade the citizens to use public mass transportation instead of their cars. We propose the use of perCues to increase the awareness for environmental pollution and to demonstrate the effect that individual actions have on the collective situation.

Ambient displays retrieve their information from a sensor network in the environment measuring among others carbon dioxide and particulate matter. The information about these emissions is visualized in the environment. Additionally, integrated mobile devices give perCues to the individual users, e.g. when a citizen leaves the house in the morning. The perCues show the positive impact of using public transport on environmental pollution compared to using a car. A user can buy a ticket either via a ticket vending machine or via mobile phone. In both cases the decrease of emissions is visualized with perCues on public displays and on the respective device (either ticket vending machine or mobile phone). In this way a user sees the direct impact of his or her individual action, in this case buying a ticket and using the bus. Additionally the individual contributions are also presented as perCues on public displays thus informing other citizens about the environmental situation. The combination of public and private perCues gives the citizens immediate positive feedback about the behavior of other people and the positive impact their own actions. This persuades them to adapt their behaviors in line with the collective goal of reducing emissions. This leads to the emergence of a collective intelligence with the goal of increased sustainability.

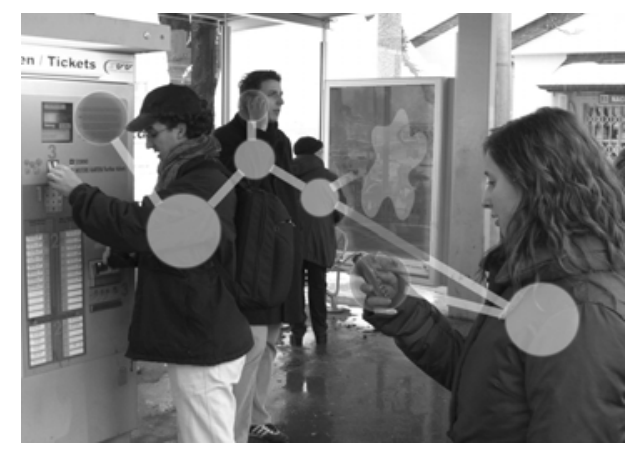

Fig. 1. Potential setting for a perCues application in a public space. The light gray circles indicate the individuals building a collective intelligence system through mutual awareness fostered by the perCues on the displays (dark gray)

The image above (Figure 1) illustrates a possible setting for our scenario in a public space. The light gray circles indicate the individuals affected by the perCues on the various displays. The displays are indicated in dark gray located on the ticket vending 
machine, mobile phones and on a large screen. The action of buying a bus ticket by the person on the left prompts a change of the perCues. They signal how much the potential use of the bus instead of a car decreases the emissions. Using perCues in public spaces we empower the citizens to reach collectively intelligent decisions.

\section{Conclusions and Future Work}

perCues derived from CI are one way to design AmI environments for persuasion. The presented scenario should demonstrate the potentials of our approach. Other examples could be persuading the members of a household to save energy, to eat healthy or to learn collectively. The examples of the tiny ants and bees can show us how to reach the goal of a global collective intelligent society. Using this approach we are able to study the effects of perCues on persuasive behavior.

\section{Acknowledgements}

This research is partially funded by the Austrian Federal Ministry for Transport, Innovation, and Technology under the FIT-IT contract FFG 809532/9295.

\section{References}

1. Weiser,M. The Computer for the Twenty-First Century. Scientific American , 94-104. 1991.

2. Aarts,E., Marzano,S.: The New Everyday: Views on Ambient Intelligence. 010 Publishers, Rotterdam (2003)

3. Lorge, I., Fox, D., Davitz, J., and Brenner, M.: A survey of studies contrasting the quality of group performance and individual performance, 1920-1957. Psychological Bulletin American Psychological Assn. 55 (1958) 337-372

4. Engelbart, D., Ruilifson, J.: Bootstrapping our collective intelligence. ACM Comput. Surv. 31, ACM Press (1999) 38

5. Lévy,P.: Collective Intelligence: Mankind's Emerging World in Cyberspace. Plenum Publishing Corp., Cambridge, MA (1997)

6. Hutchins,E.: Cognition in the Wild. MIT Press, Cambridge, Mass (1995)

7. de Ruyter, B., Saini, P., Markopoulos, P., and van Breemen, A.: Assessing the effects of building social intelligence in a robotic interface for the home. Interacting with Computers 17, 5 (2005) 522-541

8. Wehner, R.: Miniaturgehirne und kollektive Intelligenz: Zur Evolution biologischer Komplexität. Zürcher Universitätsschriften 3 (2001)

9. Lawson, J.W. and Wolpert, D.H.: The design of collectives of agents to control nonMarkovian systems. (2002) 332-337

10. Shirvanee, L. and Davenport, G.: The Viscous Display: a transient adaptive interface for collective play in public space. (2004) 259-263

11. Graggaber, M., Sperka, G.: Kyoto-Optionenbericht Salzburg. Expertenbericht der Arbeitsgruppe "Klimaschutz", Land Salzburg (2001) 